\title{
RIGHTS AND RENTS IN LOCAL COMMONS
}

\author{
Sjur Didrik Flåm
}

University of Bergen, Norway

sjur.flaameuib.no

\begin{abstract}
Motivated by management problems in national fisheries, we examine management of renewable resources in local or regional commons. This paper suggests that property rights, or lack thereof, be replaced by well-defined user rights. It shows that the use of commons can be conditioned, paid for, or valued, via market mechanisms. To that end, direct deals and double auctions are expedient. Either institution can distribute, restore and secure resource rent. Either can also focalize debates as to which assignments, regulations or taxation of rights might be fair or legitimate.
\end{abstract}

Keywords: Rights, rent, commons.

JEL Classification Numbers: C71, D33, D51, Q22.

MSC Classification: 46N10, 91A80.

\section{INTRODUCTION}

M Any commons are local or regional. Yet their governance or use is often difficult or disputed - and especially so after disruptive changes. This paper aims to address what arrangements might prove efficient, fair and stable. For a simple example, imagine an old street with no driveways or garages, where in modern times, too many cars are parked both legally or illegally. Local welfare may improve then by giving regular, specified parking permits to residents only (Fisher, 2017).

My sincere thanks go to the four anonymous referees, and my colleagues P. Bakka, I. Gaasland, R. Hannesson and B. Sandvik for their helpful feedback, and the Informatics department for excellent facilities, and Røwdes Fond for its financial support. 
This example of localized commons showcases typical characteristics. Neither use nor value of the commons is static. Indeed, either feature is much affected by changes in exogenous conditions. These facts motivate this paper to consider similar settings and inquire: can permits - and rental use of these mitigate dissipation of welfare? May well-defined user rights entail fair and legitimate sharing of surplus?

Implicitly, these questions reflect the necessity of collective action (Hughes, 1976; Olson, 1965) - or of environmental regulation and law (Fisher, 2017; Standing, 2019). ${ }^{1}$ As usual, large parts of law must be applied. In particular, the shared use of local commons ought to account for at least three defining aspects. First, throughout history, access has often depended on proximity, customary privileges or tacit conventions. Second, insiders seldom play on a level playing field with outsiders. Third, rights are not fully or approximately amended by changed abundance or use of the resources in question.

Against this backdrop, the paper considers restricted access to circumscribed commons - alongside rationed use of valuable resources therein. For an important and motivating instance, imagine a coastal region or nation that controls and determines the total quotas fishermen can catch, within its exclusive waters, during the season. ${ }^{2}$ Suppose those quotas be split according to fixed rules among legitimate parties. More precisely, suppose that qualified agents are allotted specific shares.

The privileges thus handed out are valuable allowances to "catch" specified amounts of various "species". Such allowances come as user rights, dated and with time-limited validity. Those considered here are local in nature and entail no property rights. Hence they are neither heritable nor permanently transferable (Moxnes, 2012). Yet, in the short or medium term, licensed users can ask or bid for such rights on efficient markets. That is, in the main, suppose rights are rented or leased, but not owned.

This assumption echoes Ostrom (1990) on good governance in that common resources need not be privatized. To wit, empirical studies find that agent-based exchanges and negotiations can promote efficiency and resolve conflicts; (see Chap. 3 in Ostrom, 1990). Such findings invite a search for theoretical underpinnings, and they motivate this paper to inquire: Might market-like mechanisms - operated by agents themselves - restore and safeguard substantial

1 Thereby the permits acquire economic and legal status.

2 Straddling stocks, migrating across high seas and between diverse economic zones, do not fit the frames of this paper (Ekerhovd et al., 2021). By contrast, sharing of local water does. 
parts of the resource rent? Can the distribution of realized rent also be fair and legitimate (Young, 1994)?

Each question has twin faces. One, regards institutions - as fits the resurgence of political economics (Bowles, 2004; Hodgson, 2000), (Ostrom, 1990). The other face regards solution concepts - as fits mathematical economics (Debreu, 1954, 1959), here just invoking simple convex analysis (Mordukhovich \& Nam, 2013). What combines the two faces is disequilibrium processes, mediated by stable institutions which facilitate convergence to - or emergence of - steady-state solutions. The rules remain fairly fixed, yet interactions are dynamic, open and evolutionary.

The paper is planned as follows. Section 2 provides preliminaries and briefly describes the generic agent who can use, or wants to use, the commons and resources that come with it. Section 3 depicts diverse stake-holders. Section 4 formalizes the allocation of aggregate quotas. Section 5 considers efficiency of allocations - best characterized and implemented by shadow prices. The latter define - and implicitly divide - total resource rent. Section 6 captures how shadow pricing can comply with competitive equilibrium and induce corresponding behavior (Flåm, 2020). Section 7 considers whether direct deals or double auctions can bring stakeholders to such equilibrium. Section 8 concludes.

The paper addresses the above questions asked by analysts of institutions and mechanisms, economists concerned with resource management and markets - as well as scholars of environmental law. Alongside these are computer scientists who study agent-based models. Elements of convex analysis provide the main tools.

\section{PRELIMINARIES}

Let $X$ denote a real vector space. For simplicity, $X$ has a finite dimension. Hence $X=R^{S}$ for some set $S$ denoting a finite list of "species". 3

Any vector $\left(x_{s}\right)=x \in X$ is seen as a quantified bundle of diverse natural resources, all stemming - or taken out - from some local or regional commons. By assumption, rights regarding such bundles are perfectly added, divided and transferred - without friction or transaction costs - among various identifiable and legitimate parties.

$3 x \in X$ can code a contingent claim, written on scenarios or states $s \in S$, revealed ex post. Then, if $S$ is infinite, $X$ cannot be Euclidean. Extension is possible. 
It unburdens discourse and notation to use coordinate-free expressions. Accordingly, the shorthand $x^{*} \in X^{*}$ stands for a linear functional $\hat{x} \in X \mapsto$ $x^{*} \hat{x}:=x^{*}(\hat{x}) \in R$, seen here as an arbitrage-free price regime. ${ }^{4}$ Given any gross payoff function

$$
\pi: X \mapsto R \cup\{-\infty\},
$$

such a price regime $x^{*} \in X^{*}$ is declared a supgradient of $\pi$ at $x \in X$, as is signalled by writing

$$
x^{*} \in \partial \pi(x), \text { iff } x \in \arg \max \left\{\pi(\hat{x})-x^{*} \hat{x}: \hat{x} \in X\right\} \text { with } \pi(x) \text { finite. }
$$

Thus, $x^{*} \in \partial \pi(x)$ iff the conjugate function

$$
x^{*} \in X^{*} \mapsto \pi^{*}\left(x^{*}\right):=\sup \left\{\pi(\hat{x})-x^{*} \hat{x}: \hat{x} \in X\right\}
$$

attains a finite, maximal value when $\hat{x}=x$. For interpretation: $x^{*} \in \partial \pi(x)$ iff the finite, price-taking profit $\pi^{*}\left(x^{*}\right)$ equals gross revenues $\pi(x)$ less $\cos t x^{*} x$ of input:

$$
\pi^{*}\left(x^{*}\right)=\pi(x)-x^{*} x .
$$

Some remarks on objectives follow. One may return to these - or skip them. Any gross payoff (1) should be proper, meaning that the effective domain

$$
\operatorname{dom} \pi:=\{\hat{x} \in X: \pi(\hat{x}) \in R\}=: \pi^{-1}(R)
$$

is non-empty. Typically, the set $d o m \pi$ is closed convex - often bounded whence compact - and most frequently part of $R_{+}^{S}$. To focus arguments, the implicit but rigid constraint $x \in d o m \pi$ is neither repeated nor spelled out. In short, only feasible points $x \in d o m \pi$ are considered, usually belonging to $R_{+}^{S}$. (On lack of smoothness). When payoff $\pi$ is finite near $x$ and differentiable at that point, the optimality condition (2) takes the customary form $x^{*}=\pi^{\prime}(x)$. Boundary choice in $d o m \pi$, or lack of smoothness there, cannot be ignored though. So, in the sequel, it is not necessary that payoff $\pi$ be a differentiable function.

(Linear instances). For an important example, if an agent holds resource bundle $x \in X$, acts under a linear criterion $y \in Y \mapsto y^{*} y \in R$, and uses a

4 For instance, when $X=R^{S}$, with $S$ finite, $x^{*} x=\sum_{s \in S} x_{s}^{*} x_{s}$. No arbitrage means that it is impossible to gain anything just by trading user rights. Consequently, pricing $x^{*}$ must be linear. 
resource-consuming linear technology $0 \leq y \in Y \mapsto A y \in X$, he may aim at best payoff

$$
\pi(x):=\sup \left\{y^{*} y: x \geq A y \& y \geq 0\right\} .
$$

Then, boundary or extreme point solutions $y$ become the rule. Consequently, $\pi$ is closed, ${ }^{5}$ concave and piece-wise linear, but not always differentiable or finite. ${ }^{6}$ Moreover, when $\pi(x)$ is finite,

$$
\partial \pi(x)=\arg \min \left\{x^{*} x: A^{*} x^{*} \geq y^{*} \& x^{*} \geq 0\right\}
$$

where $A^{*}: X^{*} \rightarrow Y^{*}$ denotes the transpose of $A . \diamond$

The generic agent pursues improvement or maximization of his own payoff $\pi$ (1). For analytic or technical reasons, it is convenient - in fact, desirable that any such function, as in (5), be closed concave. Further, for modelling and narrative, $\pi$ should also reflect transferable utility. How might these properties emerge? That query is addressed next.

For argument, let $X:=R \times X$ denote the augmented space of pairs $\chi=(r, x)$, each composed of monetary reserve $r \in R$ and user right $x \in X$. Suppose the agent at hand is already entitled to user right $\bar{x} \in X$. He has a preference relation $\gtrsim$ on the larger space $\mathcal{X} .^{7}$ Let $>$ denote strict preference. Given any $\chi=(r, x) \in \mathcal{X}$ in the domain of $\gtrsim$, since more money is strictly desirable, suppose

$$
\left\{\begin{array}{l}
\hat{r}>r \\
(\hat{r}, \hat{x})>(r, x) \quad \Longrightarrow(\hat{r}, x)>(r, x), \text { and } \\
(\hat{r}-\varepsilon, \hat{x})>(r, x) \text { for small enough } \varepsilon>0 .
\end{array}\right.
$$

When $\bar{\chi} \in \mathcal{X}$, let

$$
\{\gtrsim \bar{\chi}\}:=\{\chi \in \mathcal{X}: \chi \gtrsim \bar{\chi}\}
$$

denote the upper level, preferred set. Now, if the agent already holds an "initial position" $\bar{\chi}:=(\bar{r}, \bar{x}) \in \mathcal{X}$, he will pay no more money than

$$
\pi(x \mid \bar{\chi}):=\pi(x):=\sup \{r \in R:(-r, x)+\bar{\chi} \gtrsim \bar{\chi}\},
$$

5 A function $\pi: X \rightarrow R \cup\{-\infty\}$ is closed (or upper semicontinuous) iff each upper level set $\{x: \pi(x) \geq r\}, r \in R$, is closed.

${ }^{6}$ For this instance, see Flåm \& Gramstad (2012) on direct exchange in linear economies.

7 The order relation $\gtrsim$ is reflexive and transitive, but not necessarily complete. It might be represented by a utility function $U: \mathcal{X} \rightarrow R \cup\{-\infty\}$. The latter should then be closed and quasi-concave. 
for additional quota $x \in X$. Such threshold payment reflects his reservation or indifference level, conditioned by his actual endowment, holding or "wealth" $\bar{\chi}$. The function $\pi$, so defined (8), extends from $X$ to the augmented space $X=R \times X$ by

$$
\pi(\chi \mid \bar{\chi}):=\pi(\chi):=\sup \{r \in R:(-r, 0)+\chi+\bar{\chi} \gtrsim \bar{\chi}\} .
$$

Moreover, it is additively separable in money with constant slope 1 :

$$
\pi((r, 0)+\chi) \in R \Longrightarrow \pi((r, 0)+\chi)=r+\pi(\chi) .
$$

So, seen as a "commodity" which affects preferences (6), money trades at unit price 1 . Consequently,

$$
\left(r^{*}, x^{*}\right)=\chi^{*} \in \partial \pi(\chi) \text { with } \chi=(r, x) \Longleftrightarrow r^{*}=1 \& x^{*} \in \partial \pi(x) .
$$

It follows straightforwardly:

Proposition 2.1 (On Indifference Payments). If the preferred set (7) comes closed convex, the indifferent payment function $\pi$ (8) is closed concave. ${ }^{8}$

Since that function is quasi-linear in money with unit slope (9), the agent acts as though "utility" is transferable.

\section{THE AGENTS}

Fixed here is a finite ensemble $I, \# I \geq 2$, of economic agents. ${ }^{9}$ Each member $i \in I$, upon facing - and planning for - the upcoming season, considers four features of his role:

* first, he holds a right $\bar{x}_{i} \in X$ to use renewable resources found in the commons, * second, he himself chooses to take out some resource bundle $x_{i} \in X$ from there,

* third, his resulting revenue or gross payoff is determined then by a proper function

$$
x_{i} \in X \mapsto \pi_{i}\left(x_{i}\right) \in R \cup\{-\infty\},
$$

interpreted, when $x_{i} \in d o m \pi_{i}$ (4), as market income less the cost of all other factors than in bundle $x_{i}$,

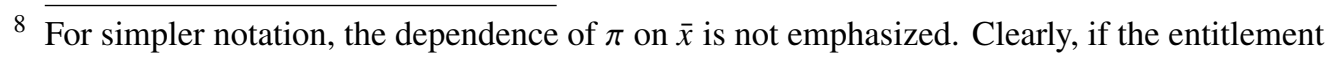
changes, so does $\pi$.

9 The setting is a closed club; nobody enters or leaves. 
* fourth, he values his excess take-out $x_{i}-\bar{x}_{i}$ by some common price $x^{*}=$ $\left(x_{s}^{*}\right) \in X^{*}$. Thus, he buys (sells) rights to take species $s \in S$ iff $x_{s}^{*}\left(x_{i s}-\bar{x}_{i s}\right)>0$ (resp. <0).

In short, agent $i$ aims at maximization of his idiosyncratic, economic criterion

$$
x_{i} \in X \mapsto \pi_{i}\left(x_{i}\right)-x^{*}\left(x_{i}-\bar{x}_{i}\right) \in R \cup\{-\infty\}
$$

(11) singles out net transaction $x_{i}-\bar{x}_{i}$ of natural input factors, valued by common price $x^{*}$.

Three types of agents merit special mention. A first, called a pure user of resources, has no established or historical rights in the commons: his $\bar{x}_{i}=0$. Yet, by money-based transactions, he acquires some user right $x_{i} \neq 0$. For example, the agent in question could be a competent fisherman, already owning fishing gear and vessel, but a priori no permit. He doesn't necessarily dwell in communities adjacent to the commons. Geographically, he might be an outsider, hence given no user right for free.

A second type $i \in I$, called a pure holder of rights, has $\bar{x}_{i} \neq 0$ but chooses $x_{i}=0$. He has no capacity - or no motivation - to use own rights himself. Instead, for suitable payments, he transfers $\bar{x}_{i}$ directly to others. Alternatively, he could put his rights up for rent, fully or partly, at various platforms for auctions or direct deals (Krishna, 2010). At those venues - or on the opposite side of markets - other parties bid for use of the rights. ${ }^{10}$ A pure holder could represent a commune, county or region which borders to - or contains - the commons. ${ }^{11}$

Third, between the said two extremes types, there are others - often many each of which has $\bar{x}_{i}$ and $x_{i}$ both non-zero. Of particular notice are those with $\bar{x}_{i}=x_{i} \neq 0$. For interpretation, these are local fishermen - active in that walk of life by long standing and tradition.

In short, three types come forward here: pure users versus pure holders of rights, and besides or between them, parties who act in both capacities. All operate within regulated, stable frames - one season after the other. Considered here is just one generic season.

\footnotetext{
${ }^{10}$ Modern versions of such platforms are computerized and accessible via the internet.

${ }^{11}$ It might be an agency, institution or syndicate.
} 


\section{SEASONAL QUOTAS}

Suppose some collective agency or directorate decides - up front, before the season - the aggregate quota $\bar{x}_{I}=\left(\bar{x}_{I S}\right) \in X=R^{S}$ to be taken, during the season, from diverse stocks $s \in S$ (Clarke, 1976). ${ }^{12}$ Presumably, that decision reflects appropriate concerns with sustainability and welfare - alongside competence in resource economics and management. ${ }^{13}$

Thereby, agent $i \in I$ is entitled to take - within specified places and times - his "fraction" $\bar{x}_{i}=\varphi_{i}\left(\bar{x}_{I}\right) \in X$ from the prescribed, total allowance $\bar{x}_{I}$, $\sum_{i \in I} \varphi_{i}\left(\bar{x}_{I}\right)=\bar{x}_{I}$. The sharing rules $\varphi_{i}: X \rightarrow X, i \in I$, are presumed fixed and constitutional in nature - continuous from one season to the next. ${ }^{14}$ What member $i \in I$ thus receives is a short-term, non-heritable user right. Suppose such rights can be renewed for free - say, annually or periodically ${ }^{15}$ - among qualified recipients.

Some remarks conclude this section. All can be skipped; none are essential.

(On dynamics and stochasticity). Cost, growth, prices or technologies can change from one season to the next. Thus, between seasons the system is dynamic and most likely stochastic. For instance, responding to environmental impacts, the resource manager may impose a seasonal moratorium on catch of different species. When the season starts, everybody perfectly understands the particular framing of the upcoming season.

(On stable frames). Interaction is governed by time-invariant, separated com-

12 The "agency" might be autonomous or independent. Presumably, it is benevolent, legitimate and respected. Other bodies - or the concerned agents themselves - ought to control compliance with rules and penalize violations.

${ }^{13}$ Stock dynamics affect seasonal quotas. Their evolution is not considered here. For this, see Bjørndal \& Conrad (1987); Clarke (1976); Gordon (1954); Hanley et al. (2007).

${ }^{14}$ By contrast, the overall abundance $\bar{x}_{I}$ may evolve from one season to the next. Innovations of various sorts, exogenous shocks and random factors drive changes in $\bar{x}_{I}$. Considered here is just one representative season. By hypothesis, the "constitution" $i \mapsto \varphi_{i}(\cdot)$ may accommodate - but hardly be overthrown by - novel regulations, states or technologies.

15 The paper side-steps these issues, simply presuming that rules $i \mapsto \varphi_{i}(\cdot)$ are given. Economic historians illuminate these issues (Libecap, 1986). It's not precluded that some rules $\varphi_{i}(\cdot)$ derive from long-term property rights. To value these - and maybe tax the proceeds - it appears important that short-term user rights be traded on well functioning markets. Suppliers could come forward fairly few - say, as municipalities, regions or the state. In any case, concerns with efficiency, fairness or taxation lend justification to establishment of markets. 
petencies and institutions - featuring a resource-managing "directorate," fixed user rights, efficient operation of auctions, direct deals or markets - and, of course, presence of "police" and possible penalties.

(On strategic behavior). Instead of individual payoff $\pi_{i}\left(x_{i}\right)$, which precludes externalities, the more general format $\pi_{i}\left(x_{i}, x_{-i}\right)$, with $x_{-i}:=\left(x_{j}\right)_{j \neq i}$, would allow congestion (or overcrowding) - and thereby some efficiency loss - in commons or markets, say of Cournot type; see Flåm (2016). This extended optic distracts, though, from the main purpose here: to identify and distribute (or restore) the fully efficient, pure resource rent. Asymmetric information - a main query in game theory (Goswami et al., 2014) - appears to have minor or negligible impacts in the present setting.

(On irreversible investments (Clarke et al., 1979)). In several industries, notably fisheries, use of natural resources requires heavy, highly specialized investment. Then, short-term user rights do not easily square with procurement of expensive, long-lived equipment. Three features may partly mitigate the problems. First, agents might enjoy perfect foresight - as is frequently assumed in market theory. Second, the said equipment is often used in other or similar contexts as well. Third, there might be - or eventually emerge - a rental market for "gear and vessels."

(On claimants to resource rent). Three parties could collect resource rent:

first, pure holders of rights, leasing these to active users,

second, active users of own rights,

third, the public sector by levying tax on quantities taken out.

Competition may eventually take pure profit away from pure users. Unlike the Malthus-Ricardo theory on income distribution between workers, merchants, and land-owners, here is scarcely any room for gentry (Dorfman, 1989) - be it coastal or landed. The basic reason is that there are no property rights in the commons.

(On matching of agents). It is conceivable - albeit not likely - that agents form a disjoint union $F \cup W=I$ of two non-empty, finite sets. Each $f \in F$ is a firm which needs input $x_{f} \in X$ but lacks user right: $\bar{x}_{f}=0$. By contrast, each $w \in W$ holds the right to use wealth $\bar{x}_{w} \in X$, but lacks capacity. Then, for stable matchings $(f, w)$, see Fujishige \& Yang (2017) and references therein. See also Ex. 259.3 in Osborne \& Rubinstein (1994). 


\section{EFFICIENCY, SHADOW PRICES, AND SHARING}

The setting is one of perfectly transferable payoffs, quotas and rights. Consequently, the convoluted criterion

$$
x_{I} \in X \mapsto \pi_{I}\left(x_{I}\right):=\sup \left\{\sum_{i \in I} \pi_{i}\left(x_{i}\right): \sum_{i \in I} x_{i}=x_{I}\right\}
$$

makes good sense - and especially so for total abundance $x_{I}=\bar{x}_{I}$. Henceforth suppose that $\pi_{I}\left(\bar{x}_{I}\right)$ be finite.

An allocation $\left(x_{i}\right)$ which solves (12) for $x_{I}=\bar{x}_{I}$ is declared efficient. Call any supgradient $x^{*} \in \partial \pi_{I}\left(\bar{x}_{I}\right)$ (2) a shadow price. Such prices support efficiency:

Proposition 5.1 (On Efficiency, Shadow Prices, and Equal Margins). Whenever $\left(x_{i}\right)$ is an efficient allocation of the aggregate $\bar{x}_{I}$, any shadow price $x^{*} \in \partial \pi_{I}\left(\bar{x}_{I}\right)$ entails common margins in that

$$
\partial \pi_{I}\left(\bar{x}_{I}\right) \subseteq \cap_{i \in I} \partial \pi_{i}\left(x_{i}\right) .
$$

Conversely, provided $\bar{x}_{I}=\sum_{i \in I} x_{i}$, the turned-around inclusion

$$
\partial \pi_{I}\left(\bar{x}_{I}\right) \supseteq \cap_{i \in I} \partial \pi_{i}\left(x_{i}\right) .
$$

also holds. If moreover, $\cap_{i \in I} \partial \pi_{i}\left(x_{i}\right)$ is non-empty, then $\left(x_{i}\right)$ is efficient.

Proof. If $x^{*} \in \partial \pi_{I}\left(\bar{x}_{I}\right)$, and $\left(x_{i}\right)$ solves (12), then $\sum_{i \in I} \hat{x}_{i}=\hat{x}_{I}$ implies

$$
\sum_{i \in I} \pi_{i}\left(\hat{x}_{i}\right) \leq \pi_{I}\left(\hat{x}_{I}\right) \leq \pi_{I}\left(\bar{x}_{I}\right)+x^{*}\left(\hat{x}_{I}-\bar{x}_{I}\right)=\sum_{i \in I}\left[\pi_{i}\left(x_{i}\right)+x^{*}\left(\hat{x}_{i}-x_{i}\right)\right] .
$$

In this string, posit $\hat{x}_{j}=x_{j}$ for each $j \neq i$ to get

$$
\pi_{i}\left(\hat{x}_{i}\right)-x^{*} \hat{x}_{i} \leq \pi_{i}\left(x_{i}\right)-x^{*} x_{i} \text { for all } \hat{x}_{i} \in X .
$$

Since $i \in I$ is arbitrary, this family of inequalities, alongside (2), implies $x^{*} \in \partial \pi_{i}\left(x_{i}\right) \forall i \in I$.

Conversely, suppose $x^{*} \in \cap_{i \in I} \partial \pi_{i}\left(x_{i}\right)$ and $\sum_{i \in I} x_{i}=\bar{x}_{I}$. Since $\pi_{i}\left(\hat{x}_{i}\right) \leq$ $\pi_{i}\left(x_{i}\right)+x^{*}\left(\hat{x}_{i}-x_{i}\right) \forall \hat{x}_{i} \in X, \forall i \in I$, summation across $I$ yields

$$
\sum_{i \in I} \pi_{i}\left(\hat{x}_{i}\right) \leq \sum_{i \in I} \pi_{i}\left(x_{i}\right)+x^{*} \sum_{i \in I}\left(\hat{x}_{i}-x_{i}\right) .
$$

Journal of Mechanism and Institution Design 5(1), 2020 
In the last inequality, let $\sum_{i \in I} \hat{x}_{i}=\bar{x}_{I}$ to see that allocation $\left(x_{i}\right)$ solves (12). Further, the instance $\sum_{i \in I} \hat{x}_{i}=\hat{x}_{I}$ entails $\pi_{I}\left(\hat{x}_{I}\right) \leq \pi_{I}\left(\bar{x}_{I}\right)+x^{*}\left(\hat{x}_{I}-\bar{x}_{I}\right) \forall \hat{x}_{I} \in X$. Hence $x^{*} \in \partial \pi_{I}\left(\bar{x}_{I}\right)$.

Proposition 5.1 invokes no assumptions - apart, of course, from availability of suitable allocations $\left(x_{i}\right)$ and prices $x^{*}$. Existence of such "items" is briefly addressed next so as to "reduce" or play down the role of convexity:

Proposition 5.2 (On the Existence of Price-Supported Pareto Optimum). Suppose that each criterion $\pi_{i}$ (10) be proper and sup-compact. ${ }^{16}$ Then the optimal value $\pi_{I}\left(\bar{x}_{I}\right)$ (12) is attained.

If convolution $\pi_{I}(12)$ is bounded below near $\bar{x}_{I}$ and coincides at that point with its closed concave envelope, then $\partial \pi_{I}\left(\bar{x}_{I}\right)$ is non-empty.

Proof (sketched). One argues directly that $\pi_{I}$ (12) inherits concavity from its underlying terms $\pi_{i}$ - and likewise for having $\pi_{I}$ closed. By compactness, the latter property secures that $\pi_{I}\left(\bar{x}_{I}\right)$ is attained. Finally, any closed concave function, bounded below near $\bar{x}_{I}$, has non-empty supdifferential $\partial \pi_{I}\left(\bar{x}_{I}\right)$ (Zălinescu, 2002).

A running example accommodates just one species: $\# S=1$ so that $X=R$. Suppose agent $i \in I$ has catch capacity $\kappa_{i} \geq 0$ and earns gross revenue $r_{i} \geq 0$ per unit caught. Then, $\pi_{i}\left(x_{i}\right)=r_{i} x_{i}$ if $x_{i} \in\left[0, \kappa_{i}\right]=d o m \pi_{i}(4)-$ and $-\infty$ elsewhere. So,

$$
\partial \pi_{i}\left(x_{i}\right)= \begin{cases}\left(+\infty, r_{i}\right] & \text { if } x_{i}=0, \\ r_{i} & \text { if } 0<x_{i}<\kappa_{i}, \\ {\left[r_{i},-\infty\right)} & \text { if } x_{i}=\kappa_{i}\end{cases}
$$

and $\partial \pi_{i}\left(x_{i}\right)$ is empty otherwise. Let $I:=\{1, \ldots, \# I\}$ and suppose $r_{1}>r_{2}>\cdots$. For specified aggregate quota $q:=\bar{x}_{I}>0$, define $i(q)$ to be the smallest index $i \in I$, if any, such that

$$
\sum\left\{\kappa_{i}: i \leq i(q)\right\} \geq q .
$$

Provided $i(q)$ is well defined, the efficient allocation $\left(x_{i}\right)$ has $x_{i}=\kappa_{i}$ for each intra-marginal $i<i(q)$. The marginal agent $i(q)$ gets what remains of the total quota. For simplicity in argument, suppose his allotment is strictly interior to

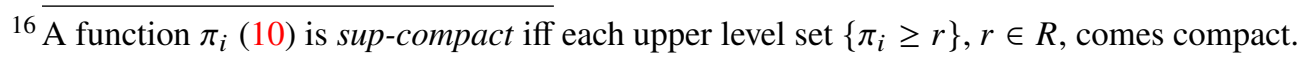


the interval $\left[0, \kappa_{i(q)}\right]$. Every extra-marginal agent $i>i(q)$ gets $x_{i}=0$. Thus, each agent $i$, but at most one, ends up with boundary choice $x_{i} \in\left\{0, \kappa_{i}\right\}$.

By Proposition 5.1 and (14), user rights trade at unit price $x^{*}=r_{i(q)} \in$ $\cap_{i \in I} \partial \pi_{i}\left(x_{i}\right)$. Agent $i$ earns profit

$$
\pi_{i}\left(x_{i}\right)-x^{*}\left(x_{i}-\bar{x}_{i}\right)= \begin{cases}r_{i} \kappa_{i}-r_{i(q)}\left(\kappa_{i}-\bar{x}_{i}\right) & \text { if } i<i(q), \\ r_{i} x_{i}-r_{i(q)}\left(x_{i}-\bar{x}_{i}\right) & \text { if } i=i(q) \\ r_{i(q)} \bar{x}_{i} & \text { if } i>i(q)\end{cases}
$$

In the unlikely event that $\sum_{i \in I} \kappa_{i}<q:=\bar{x}_{I}$, user rights are worthless: $x^{*}=0 . \diamond$

Price-supported allocations are Pareto optimal. They also stand out on other grounds - as is described in the next section.

\section{EQUILIBRIUM AND WELFARE}

Proposition 5.1 revolves around coincidence of marginal valuations. That feature - combined with price-taking and optimizing behavior - characterizes competitive equilibria:

Definition 6.1 (Competitive Equilibrium in User Rights). An allocation cum price pair $\left[\left(x_{i}\right), x^{*}\right] \in X^{I} \times X^{*}$ constitutes a price-taking equilibrium iff

$$
\begin{cases}\text { quota markets clear: } & \sum_{i \in I} x_{i}=\bar{x}_{I}, \\ \text { with best choices: } & \pi_{i}\left(x_{i}\right)-x^{*} x_{i}=\max \left\{\pi_{i}-x^{*}\right\} \in R \forall i \in I .\end{cases}
$$

Proposition 6.1 (On Competitive Equilibrium, Profit and Sharing of Resource Rent). If agent $i \in I$ regards the price $x^{*} \in X^{*}$ of quotas as exogenous, he may aim at the highest profit

$$
\pi_{i}^{*}\left(x^{*}\right):=\sup \left\{\pi_{i}\left(\hat{x}_{i}\right)-x^{*} \hat{x}_{i}: \hat{x}_{i} \in X\right\} .
$$

Provided $\pi_{i}(0) \geq 0$, that profit is non-negative. Moreover, for any shadow price $x^{*} \in \partial \pi_{I}\left(\bar{x}_{I}\right)$ and allocation $\left(x_{i}\right)$ which solves $(12)$ with $x_{I}=\bar{x}_{I}$, agent $i$ can take home the highest total payment

$$
\Pi_{i}\left(x^{*}\right):=\pi_{i}^{*}\left(x^{*}\right)+x^{*} \bar{x}_{i}=\left[\pi_{i}\left(x_{i}\right)-x^{*} x_{i}\right]+x^{*} \bar{x}_{i},
$$

composed of pure profit $\pi_{i}^{*}\left(x^{*}\right) \geq 0$, derived from production, plus resource rent $\rho_{i}:=x^{*} \bar{x}_{i}$ from own entitlement. In particular, a pure resource user $i$ just 
collects production profit $\pi_{i}^{*}\left(x^{*}\right)$. At the other extreme, a pure right holder $i$ merely receives his part $\rho_{i}=x^{*} \bar{x}_{i}$ of the total resource rent $\rho_{I}:=x^{*} \bar{x}_{I}{ }^{17}$

Proof. (15) is a matter of definition and price-taking behavior. Clearly $\pi_{i}^{*}\left(x^{*}\right) \geq \pi_{i}(0)-x^{*} 0 \geq 0$. When $\left(x_{i}\right)$ solves (12) for $x_{I}=\bar{x}_{I}$, and $x^{*} \in \partial \pi_{I}\left(\bar{x}_{I}\right)$, it follows from (13) that $x^{*} \in \partial \pi_{i}\left(x_{i}\right)$. Hence maximal profit (15) is attained with input $x_{i}$ (2). So, net value (16) follows.

As is well known, competitive equilibrium connects to Pareto efficiency via two fundamental welfare theorems (Luenberger, 1995). Those connections are applicable here. At the same time, incentives and stability come to the fore. To wit, consider a transferable-payoff, cooperative game with player set I in which any non-empty coalition $\mathcal{I} \subseteq I$ could aim for a joint payoff

$$
\pi_{\mathcal{I}}\left(\bar{x}_{\mathcal{I}}\right):=\sup \left\{\sum_{i \in \mathcal{I}} \pi_{i}\left(x_{i}\right): \sum_{i \in \mathcal{I}} x_{i}=\bar{x}_{\mathcal{I}}:=\sum_{i \in \mathcal{I}} \bar{x}_{i}\right\} .
$$

Then, a payoff profile $i \in I \mapsto \Pi_{i} \in R$ belongs to the core (Osborne \& Rubinstein, 1994) iff

$$
\sum_{i \in \mathcal{I}} \Pi_{i} \geq \pi_{\mathcal{I}}\left(\bar{x}_{\mathcal{I}}\right) \quad \forall \mathcal{I} \subseteq I \text { with equality for the grand coalition } \mathcal{I}=I .
$$

Proposition 6.2 (On Price-Supported Payoff-Sharing and Core Solutions). For any $x^{*} \in X^{*}$ the payment profile $i \in I \mapsto \Pi_{i}:=\pi_{i}^{*}\left(x^{*}\right)+x^{*} \bar{x}_{i}$ satisfies the inequalities in (17). Moreover, equality holds for the grand coalition $\mathcal{I}=I$ iff $x^{*} \in \partial \pi_{I}\left(\bar{x}_{I}\right)$. Thus, any shadow price $x^{*}$, alongside any best allocation $\left(x_{i}\right)$ of $\bar{x}_{I}$ across $I$, implements a core solution in which agent $i$ gets $\Pi_{i}=\Pi_{i}\left(x^{*}\right)$ (16).

Conversely, if $x_{i}$ solves (15) and $\sum_{i \in I} x_{i}=\bar{x}_{I}$, then $\left(x_{i}\right)$ is a best allocation of $\bar{x}_{I}$. Further $x^{*} \in \partial \pi_{I}\left(\bar{x}_{I}\right)$, and the profile $i \mapsto \Pi_{i}\left(x^{*}\right)$ (16) is "in the core" (17).

Proof. For any non-empty $\mathcal{I} \subseteq I$ and price $x^{*} \in X^{*}$, inequality (17) holds

${ }^{17}$ Some goal or golden rule (Clarke, 1976) might define a steady yield vector $\bar{x}_{I} \in X$ alongside a shadow price $x^{*} \in \partial \pi_{I}\left(\bar{x}_{I}\right)$. Regarding the components of $\bar{x}_{I}$ as material dividends, furnished by nature, $x^{*} \bar{x}_{I}$ equals the total resource rent. Absent perfect markets, resource pricing is difficult (Neher, 1990). Formula $x^{*} \bar{x}_{I}$ fits, however, competitive economies in which production factors are paid according to their marginal values. 
because

$$
\begin{aligned}
\sum_{i \in \mathcal{I}} \Pi_{i}\left(x^{*}\right) & \geq \sup \left\{\sum_{i \in \mathcal{I}}\left[\pi_{i}\left(x_{i}\right)+x^{*}\left(\bar{x}_{i}-x_{i}\right)\right]:\left(x_{i}\right) \in X^{\mathcal{I}}\right\} \\
& \left.\geq \sup \left\{\sum_{i \in \mathcal{I}} \pi_{i}\left(x_{i}\right)+x^{*}\left(\bar{x}_{i}-x_{i}\right)\right]: \sum_{i \in \mathcal{I}} x_{i}=\bar{x}_{\mathcal{I}}\right\}=\pi_{\mathcal{I}}\left(\bar{x}_{\mathcal{I}}\right) .
\end{aligned}
$$

In particular, $\sum_{i \in I} \Pi_{i}\left(x^{*}\right) \geq \pi_{I}\left(\bar{x}_{I}\right)$. For the converse of the last inequality, note that $x^{*} \in \partial \pi_{I}\left(\bar{x}_{I}\right)$ and $\sum_{i \in I} x_{i}=x_{I}$ imply $\sum_{i \in I} \pi_{i}\left(x_{i}\right) \leq \pi_{I}\left(x_{I}\right) \leq \pi_{I}\left(\bar{x}_{I}\right)+$ $x^{*}\left(x_{I}-\bar{x}_{I}\right)$, whence

$$
\sum_{i \in I}\left[\pi_{i}\left(x_{i}\right)+x^{*}\left(\bar{x}_{i}-x_{i}\right)\right] \leq \pi_{I}\left(\bar{x}_{I}\right) \forall\left[x_{i}\right] \in X^{I} .
$$

In (18) take supremum over profiles $\left(x_{i}\right)$ to see that $\sum_{i \in I} \Pi_{i}\left(x^{*}\right) \leq \pi_{I}\left(\bar{x}_{I}\right)$. Inequality (18) also implies that $x^{*} \in \partial \pi_{I}\left(\bar{x}_{I}\right)$. Finally, Proposition 5.1 justifies the claims about attainment of core payments.

Remarks (on competitive equilibrium). The solution concept in Def. 6.1, is Walrasian (Debreu, 1959). It implies, strictly speaking, that all trade takes place once, in equilibrium - at common, clearing prices. This framing begs two reservations. First, most likely - since data may change from one season to another - equilibrium becomes contingent, depending on exogenous factors. This issue is addressed later by emphasizing that Debreu's concept of valuation equilibrium (Debreu, 1954) appears more fitting ; see remark after Proposition 7.4 .

Second, the Walrasian setting precludes that rights be traded out of equilibrium, prior to the season, maybe repeatedly and at "personal" prices - as will be considered next.

\section{RIGHTS TRADED}

How might equilibrium come about? In what manner could clearing prices emerge ${ }^{18}$ To address these questions, this section considers two market mechanisms, namely: direct deals and double auctions - in that order.

18 Walrasian tâtonnement might illuminate matters, but isn't practical; see Flåm (2020) and the agent-based approach (Bowles, 2004). 
Direct deals (Flåm, 2014) are decentralized and iterative. For a backdrop, invoking shadow prices $x^{*} \in \cap_{i \in I} \partial \pi_{i}\left(x_{i}\right)$, Proposition 5.1 captures and describes efficient outcomes. Otherwise, if $\cap_{i \in I} \partial \pi_{i}\left(x_{i}\right)$ is empty, then already $\cap_{i \in \mathcal{I}} \partial \pi_{i}\left(x_{i}\right)=\varnothing$ for some subset $\mathcal{I} \subseteq I$, comprising at least two agents. In this case, resource valuations differ among members of $\mathcal{I}$; that is, some bid-ask spread prevails across $\mathcal{I}$.

That concept, is formally defined next and generalized - beyond price difference in one good - to multi-commodity settings. Recall that the directional derivative of a concave $\pi_{i}(\cdot)$ at $x_{i}$ - with $\pi_{i}\left(x_{i}\right)$ finite - in direction $d_{i}$ is given by

$$
\pi_{i}^{\prime}\left(x_{i} ; d_{i}\right)=\lim _{r \rightarrow 0^{+}} \frac{\pi_{i}\left(x_{i}+r d_{i}\right)-\pi_{i}\left(x_{i}\right)}{r} .
$$

Hereafter, for each $i \in I$, suppose $\pi_{i}^{\prime}\left(x_{i} ; d_{i}\right)$ is closed in $d_{i}$. Then,

$$
\pi_{i}^{\prime}\left(x_{i} ; d_{i}\right)=\inf \left\{x_{i}^{*} d_{i}: x_{i}^{*} \in \partial \pi_{i}\left(x_{i}\right)\right\} .
$$

Definition 7.1 (Bid-Ask Spread). Let $\|\cdot\|$ denote the norm on $X$. An agent ensemble $\mathcal{I} \subseteq I, \# \mathcal{I} \geq 2$, which actually holds a profile $i \in \mathcal{I} \mapsto x_{i} \in X$, displays bid-ask spread

$$
\mathfrak{S}_{\mathcal{I}}(\mathbf{x}):=\max _{\left(d_{i}\right)}\left\{\sum_{i \in \mathcal{I}} \pi_{i}^{\prime}\left(x_{i} ; d_{i}\right): \sum_{i \in \mathcal{I}} d_{i}=0, \sum_{i \in \mathcal{I}}\left\|d_{i}\right\|^{2} \leq 1\right\} .
$$

Remark (on bid-ask spread). Suppose (19) is applicable. Then, invoke Theorem 1.86 in (Penot, 2013) to replace the resulting max inf format (20) with inf max. Thus,

$$
\mathfrak{S}_{\mathcal{I}}(\mathbf{x})=\inf _{\left(x_{i}^{*}\right)} \max _{\left(d_{i}\right)}\left\{\sum_{i \in \mathcal{I}} x_{i}^{*} d_{i}: x_{i}^{*} \in \partial \pi_{i}\left(x_{i}\right) \& \sum_{i \in \mathcal{I}} d_{i}=0, \quad \sum_{i \in \mathcal{I}}\left\|d_{i}\right\|^{2} \leq 1\right\} .
$$

Here, the operation $\max _{\left(d_{i}\right)}$ is easily performed. Indeed, using shorthand $\bar{x}^{*}:=$ $\sum_{i \in \mathcal{I}} x_{i}^{*} / \# \mathcal{I}$ for the average of supgradients $x_{i}^{*} \in \partial \pi_{i}\left(x_{i}\right), i \in \mathcal{I}$, when some $x_{i}^{*} \neq \bar{x}^{*}$, yields

$$
\mathfrak{S}_{\mathcal{I}}(\mathbf{x})=\min \left\{\left[\sum_{i \in \mathcal{I}}\left\|x_{i}^{*}-\bar{x}^{*}\right\|^{2}\right]^{-1 / 2} \sum_{i \in \mathcal{I}} x_{i}^{*}\left(x_{i}^{*}-\bar{x}^{*}\right): x_{i}^{*} \in \partial \pi_{i}(x)\right\} . \diamond
$$


As indicated, a positive spread signals that agents' valuations diverge:

Proposition 7.2 (On Bid-Ask Spreads). Bid-ask spread (20) is non-negative: $\Im_{I}(\mathbf{x}) \geq 0$. Moreover, with each $\partial \pi_{i}\left(x_{i}\right)$ non-empty compact, then

$$
\mathfrak{S}_{I}(\mathbf{x})=0 \Longleftrightarrow \cap_{i \in I} \partial \pi_{i}\left(x_{i}\right) \neq \varnothing .
$$

Proof. The simple fact that each $\pi_{i}^{\prime}\left(x_{i} ; 0\right)=0$ implies $\mathfrak{S}_{\mathcal{I}}(\mathbf{x}) \geq 0$. Further, for any $x^{*} \in \cap_{i \in \mathcal{I}} \partial \pi_{i}\left(x_{i}\right)$,

$$
\mathfrak{S}_{\mathcal{I}}(\mathbf{x}) \leq \max \left\{\sum_{i \in \mathcal{I}} x^{*} d_{i}: \sum_{i \in \mathcal{I}} d_{i}=0, \sum_{i \in \mathcal{I}}\left\|d_{i}\right\|^{2} \leq 1\right\}=0,
$$

in which case, $\mathfrak{S}_{\mathcal{I}}(\mathbf{x})=0$. Conversely, when each $\partial \pi_{i}\left(x_{i}\right)$ is non-empty and compact but $\cap_{i \in \mathcal{I}} \partial \pi_{i}\left(x_{i}\right)=\varnothing$, use a non-zero vector $\mathbf{d}=\left(d_{i}\right) \in X^{\mathcal{I}}$ to separate the compact convex product set $\Pi_{i \in \mathcal{I}} \partial \pi_{i}\left(x_{i}\right)$ strictly from the "diagonal" $\left\{\left(x_{i}^{*}\right):\right.$ all $x_{i}^{*}$ are equal $\}$. Then, $\sum_{i \in \mathcal{I}} d_{i}=0$, and one may take $\sum_{i \in \mathcal{I}}\left\|d_{i}\right\|^{2}=1$. Finally, by choosing the appropriate sign of $\mathbf{d}$, it follows that $\mathfrak{S}_{\mathcal{I}}(\mathbf{x})>0$.

In the running example (see Sect. 5), when $x_{1} \in\left[0, \kappa_{1}\right)$ and $x_{2} \in\left(0, \kappa_{2}\right]$, agent ensemble $\mathcal{I}=\{1,2\}$ has spread $\mathfrak{S}_{\mathcal{I}}(\mathbf{x})=r_{1}-r_{2}>0$. Further, the overall spread $\mathfrak{\Im}_{I}(\mathbf{x})$ is nil iff $r_{i(q)}=\cap_{i \in I} \partial \pi_{i}\left(x_{i}\right) . \diamond$

When $\mathfrak{S}_{\mathcal{I}}(\mathbf{x})>0$, members of $\mathcal{I}$ have good reasons to transact among themselves. Clearly, direct deals within $\mathcal{I}$ are purely redistributive. So, with no loss of generality, agent $i \in \mathcal{I}$ gets an updated user right

$$
x_{i}^{+1}:=x_{i}+\sigma d_{i} \text { with } \sum_{i \in \mathcal{I}} d_{i}=0 \text { and } \sum_{i \in \mathcal{I}}\left\|d_{i}\right\|^{2} \leq 1
$$

for some step-size $\sigma \geq 0$. It's assumed that $\mathbf{x}^{+1}:=\left(x_{i}^{+1}\right)$, so defined with $x_{i}^{+1}=x_{i}$ for $i \notin \mathcal{I}$, remains feasible, meaning $x_{i}^{+1} \in d o m \pi_{i}$ - that is, $\pi_{i}\left(x_{i}^{+1}\right) \in R$ for $i \in \mathcal{I}$.

Assumption (On Payoff Improvements). Whenever agents $i \in \mathcal{I} \subseteq I, \# \mathcal{I} \geq 2$, are trading user rights among themselves, redistribution (23) entails total payoff improvement

$$
\Delta \pi_{\mathcal{I}}(\mathbf{x}):=\sum_{i \in \mathcal{I}}\left[\pi_{i}\left(x_{i}^{+1}\right)-\pi_{i}\left(x_{i}\right)\right] \geq \sigma \widetilde{\Xi}_{\mathcal{I}}(\mathbf{x})
$$


for some "step-size" $\sigma>0$, depending on the stage counter $k$ - or the number of encounters, so far, at which I traded.

Repeated direct deals are modelled next as a discrete-time process (23) in the nature of an algorithm:

Start, with $x_{i}=\bar{x}_{i}$ for each $i \in I$.

Activate, by some protocol or matching mechanism, an agent ensemble $\mathcal{I} \subseteq I$, $\# I \geq 2$.

If $\mathfrak{S}_{I}(\mathbf{x})>0$, its members trade among themselves so that (23) yields (24).

Continue to Activate some agent ensemble until convergence.

To repeat: at discrete stages $k=0,1, \ldots$ user right $x_{i}^{k}$ for $i \in \mathcal{I}^{k} \subseteq I$ is updated by

$$
x_{i}^{k+1}:=x_{i}^{k}+\sigma^{k} d_{i}^{k} \text { with } \sum_{i \in I^{k}} d_{i}^{k}=0 \text { and } \sum_{i \in \mathcal{I}^{k}}\left\|d_{i}^{k}\right\|^{2} \leq 1
$$

Henceforth suppose step-sizes dwindle, meaning $\sigma^{k} \rightarrow 0^{+}$.

The resulting process depends, of course, on which ensemble $\mathcal{I}^{k} \subseteq I$ of agents trade at stage $k$. In particular, it appears prudent that ensembles come forward almost periodically:

Proposition 7.3 (On Eventual Disappearance of Bid-Ask Spreads). Let the members of some ensemble $\mathcal{I} \subseteq I, \# \mathcal{I} \geq 2$, trade repeatedly among themselves (23), with no less than a fixed, finite lapse between consecutive stages. Suppose step-sizes dwindle in that $\sigma^{k} \rightarrow 0^{+}$, but not too fast, meaning that for any subsequence $\mathbf{x}^{k}, k \in K$, along which $\mathcal{I}$ trades,

$$
\mathbf{x}=\lim _{k \in K} \mathbf{x}^{k} \& \mathfrak{S}_{\mathcal{I}}(\mathbf{x})>0 \Longrightarrow \sum_{k \in K} \sigma^{k}=+\infty
$$

Then, for any subsequential limit $\mathbf{x}=\lim _{k \in K} \mathbf{x}^{k}$, it holds $\mathfrak{S}_{I}(\mathbf{x})=0$.

Proof. Consider any subsequential limit $\mathbf{x}=\lim _{k \in K} \mathbf{x}^{k}$. Since $\sigma^{k} \rightarrow 0^{+}$, and the ensemble $\mathcal{I}$ comes on stage almost cyclically, it brings no loss of generality to assume that the members of $\mathcal{I}$ trade (among themselves) at all 
stages $k \in K$. Now, if $\mathfrak{S}_{\mathcal{I}}(\mathbf{x})>0$, invoke (25) to see that

$$
\begin{aligned}
+\infty & >\pi_{I}(\bar{x})-\sum_{i \in I} \pi_{i}\left(\bar{x}_{i}\right) \geq \lim _{k \rightarrow+\infty} \sum_{i \in I} \pi_{i}\left(x_{i}^{k+1}\right)-\sum_{i \in I} \pi_{i}\left(\bar{x}_{i}\right) \\
& \geq \sum_{k \in K} \sum_{i \in \mathcal{I}}\left[\pi_{i}\left(x_{i}^{k+1}\right)-\pi_{i}\left(x_{i}^{k}\right)\right] \geq \sum_{k \in K} \Delta \pi_{\mathcal{I}}\left(\mathbf{x}^{k}\right) \geq \sum_{k \in K} \sigma^{k} \Im_{I}(\mathbf{x})=+\infty .
\end{aligned}
$$

Declare a family $\mathbb{I}$ of subsets from $I$ decisive if

$$
\mathfrak{S}_{\mathcal{I}}(\mathbf{x})=0 \forall \mathcal{I} \in \mathbb{I} \Rightarrow \mathfrak{S}_{I}(\mathbf{x})=0 .
$$

Two examples: (I) If some $i \in I$ has differentiable payoff $\pi_{i}$ and I comprises all agent pairs $\{i, j \neq i\}$, the family $\mathbb{I}$ is decisive. This setting indicates that some "smooth" agent $i$ serves as a hub for trade.

(II) Suppose I comprises all ensembles $\mathcal{I} \subseteq I$ with $\# \mathcal{I}=\# S+1$, and let each be $\partial \pi_{i}\left(x_{i}\right)$ compact. Then, since $X=R^{S}$, by Helly's theorem (Valentine, 1964), II becomes decisive. $\diamond$

Proposition 6.3 immediately implies:

Corollary to Propostion 7.3 (On Competitive Equilibria as Cluster Points). If the members of each ensemble I, belonging to a decisive family II (26), trade at least once between themselves within any lapse of some prescribed length, then for every accumulation point $\mathbf{x}$ of traded holdings $\mathbf{x}^{k}, k=0,1, \ldots$ it holds $\mathfrak{S}_{I}(\mathbf{x})=0$. If moreover, all $\partial \pi_{i}\left(x_{i}\right)$ are non-empty compact at such a point $\mathbf{x}=\left(x_{i}\right)$, there exists a price $x^{*} \in X^{*}$ such that $\left[\left(x_{i}\right), x^{*}\right]$ is a competitive equilibrium.

Direct trade unfolds as a discrete-time process. Most exchanges happen off equilibrium, and limits depend on sequential matchings of agents.

Considered next is a markedly different institution, called double auction. Trade takes place indirectly at such venues, once, and only in equilibrium chiefly depending on endowments:

The double auction (Flåm, 2021) is a one-shot affair. Each participant $i \in I$ anonymously, "simultaneously" and silently submits his payoff function $\pi_{i}(\cdot)$ - say, "in closed form", in "a sealed envelope" - to a common auctioneer. Thereby, qua "bidder", agent $i$ commits to "buy" whatever user right $x_{i} \in X$ for whatever payment $\leq \pi_{i}\left(x_{i}\right)$. 
The auctioneer convolutes the received functions $\pi_{i}(\cdot), i \in I$, so as to solve (12), when $x_{I}=\bar{x}_{I}$, for an optimal allocation $\left(x_{i}\right)$, supported by some shadow price $x^{*} \in \partial \pi_{I}\left(\bar{x}_{I}\right)$. Thereafter, he allots user right $x_{i}$ to agent $i$ for net payment $x^{*}\left(x_{i}-\bar{x}_{i}\right)$. Thus, the auction house clears. Aggregate demand equals total supply: $\sum_{i \in I} x_{i}=\bar{x}_{I}$. The auctioneer merely redistributes seasonal endowments $\bar{x}_{i}$ and "charges" payments $x^{*}\left(x_{i}-\bar{x}_{i}\right)$ for "excess use". ${ }^{19}$ Proposition 5.1 immediately entails:

Proposition 7.4 (Redistribution via a Double Auction). Suppose no party $i \in I$ behaves strategically but rather submits his function $\pi_{i}(\cdot)$ to the auctioneer as is. Also suppose the latter solves instance $\pi_{I}\left(\bar{x}_{I}\right)$ (12) for an efficient allocation $\left(x_{i}\right)$, supported by a shadow price $x^{*} \in \partial \pi_{I}\left(\bar{x}_{I}\right)$. Thereby, the auctioneer implements a competitive equilibrium $\left[\left(x_{i}\right), x^{*}\right]$ in user rights.

Remarks: (On double auctions). As modelled, the double auction relates to Walrasian tâtonnement. It is markedly different though. The auctioneer of Walras pushes a price process so as to equilibrate intended demand with supply. The double auction is no process; it convolutes price-quantity curves to have one-shot clearing. Such institutions weaken the customary criticism of the Walrasian paradigm.

However, since payoff $\pi_{i}\left(\cdot \mid \bar{\chi}_{i}\right)$ going to any party $i$ depends on his endowment $\bar{\chi}_{i}=\left(\bar{r}_{i}, \bar{x}_{i}\right)$, there are non-standard wealth effects. Specializing Definition 6.1 allows such effects to be incorporated: Following Debreu (Debreu, 1954), a valuation equilibrium $\left[x^{*},\left(x_{i}\right)\right]$ prevails iff

$$
\begin{cases}\text { markets clear: } & \sum_{i \in I} x_{i}=\bar{x}_{I}, \\ \text { with no pure profit: } & \pi_{i}\left(x_{i}\right)-x^{*} x_{i}=\max \left\{\pi_{i}-x^{*}\right\}=0 \forall i \in I .\end{cases}
$$

Then, for any agent $i \in I$, if he is a consumer, $\hat{x}_{i}>_{i} x_{i} \Longrightarrow x^{*} \hat{x}_{i}>x^{*} x_{i}$. If he is a producer, $x^{*} \hat{x}_{i}>x^{*} x_{i} \Longrightarrow \hat{x}_{i}$ is infeasible; see Flåm (2021). With this solution concept, $i$ takes home pure resource rent $x^{*} \bar{x}_{i}$ and no more.

Notice though, that the customary distinction between consumers and producers does not apply well in commons.

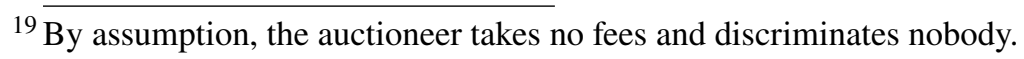




\section{CONCLUDING REMARKS}

Management of local commons and resources brings up important concerns (Ostrom, 1990). Many revolve around how to "get institutions right." This paper advocates stable rules and separation of powers. Three major points stand out:

First, an independent bio-economic agency ought, qua quantity adjustor, to care about the aggregate take and the intertemporal allocation of resources, thereby mitigating negative externalities and blocking tragedies of the commons (Hardin, 1968). ${ }^{20}$

Second, for efficiency ${ }^{21}$ and fairness, the paper recommends that user rights should replace or supplement property rights. ${ }^{22}$ Under fair sharing, a rental market might enhance efficiency and eventually meet with common acceptance.

Third, there is no escape from strict rules, tight control and suitable penalties.

\section{References}

Bjørndal, T., \& Conrad, J. M. (1987). The dynamics of an open access fishery. Canadian Journal of Economics, 20, 74-85.

Bowles, S. (2004). Microeconomics. Princeton University Press.

Clarke, C. W. (1976). Mathematical bioeconomics. J. Wiley \& Sons, New York.

Clarke, C. W., Clarke, F. H., \& Munro, G. R. (1979). The optimal exploitation of renewable resource stocks: Problems of irreversible investment. Econometrica, 47(1), 25-47.

Debreu, G. (1954). Valuation equilibrium and Pareto optimum. Proceedings of the National Academy of Sciences, 47(1), 588-92.

Debreu, G. (1959). Theory of value. Yale University Press, New Haven.

Dorfman, R. (1989). Thomas Robert Malthus and David Ricardo. Journal of Economic Perspectives, 3(3), 153-64.

Ekerhovd, N.-A., Flåm, S. D., \& Steinshamn, S. I. (2021). On shared use of straddling stocks. European Journal of Operations Research, forthcoming.

${ }^{20}$ One can hardly expect much constructive logic of collective action (Olson, 1965) among parties who have more of opposed than of common interest.

${ }^{21}$ It requires no justification to emphasize the welfare gains generated by efficient use of scarce production factors (Kaldor, 1939).

${ }^{22}$ Shifting parts of resource rent - away from users of rights - towards permanent holders of such items, is apt to provoke contestation. 
Fisher, E. (2017). Environmental law. Oxford University Press.

Flåm, S. D. (2014). Bilateral exchange and competitive equilibrium. Set-Valued and Variational Analysis, 24, 1-11.

Flåm, S. D. (2016). Noncooperative games, coupling constraints, and partial efficiency. Bulletin of Economic Theory, 4, 213-29.

Flåm, S. D. (2020). Emergence of price-taking behavior. Economic Theory, 70, 847-70.

Flåm, S. D. (2021). Towards competitive equilibrium by double auctions. Pure and Applied Functional Analysis, forthcoming.

Flåm, S. D., \& Gramstad, K. (2012). Direct exchange in linear economies. International Game Theory Review, 14(4), 1-18.

Fujishige, S., \& Yang, Z. (2017). On a spontaneous decentralized market process. Journal of Mechanism and Institution Design, 2, 1-37.

Gordon, H. S. (1954). The economic theory of a common property resource: The fishery. Journal of Political Economy, 62, 124-42.

Goswami, M. P., Mitra, M., \& Sen, A. (2014). Strategy proofness and Pareto efficiency in quasilinear exchange economies. Theoretical Economics, 9, 361-81.

Hanley, N., Shogren, J. F., \& White, B. (2007). Environmental economics (2nd ed.). Palgrave, New York.

Hardin, G. (1968). The tragedy of the commons. Science, 162, 143-48.

Hodgson, G. M. (2000). What is the essence of institutional economics? Journal of Economic Issues, 34, 317-29.

Hughes, J. R. T. (1976). Social control in the colonial economy. University of Virgina Press, Charlottesville.

Kaldor, N. (1939). Welfare propositions of economics and interpersonal comparisons of utility. Economic Journal, 49, 549-52.

Krishna, V. (2010). Auction theory. Academic Press, Amsterdam.

Libecap, G. D. (1986). Property rights in economic history: Implications for research. Explorations in Economic History, 23, 227-52.

Luenberger, D. G. (1995). Microeconomic theory. McGraw-Hill, New York.

Mordukhovich, B. S., \& Nam, N. M. (2013). An easy path to convex analysis and applications. Morgan \& Claypool Publishers.

Moxnes, E. (2012). Individual transferable quotas versus auctioned seasonal quotas: An experimental investigation. Marine Policy, 36, 339-49.

Neher, P. A. (1990). Natural resource economics, conservation and exploitation. Cambridge University Press.

Olson, M. (1965). The logic of collective action, public goods, and the theory of groups. Harvard University Press.

Osborne, M. J., \& Rubinstein, A. (1994). A course in game theory. MIT Press. 
Ostrom, E. (1990). Governing the commons. Cambridge University Press.

Penot, J.-P. (2013). Calculus without derivatives. Springer Verlag, Berlin.

Standing, G. (2019). Plunder of the commons. Pelican Books, Random House, UK.

Valentine, F. A. (1964). Convex sets. Mc-Graw-Hill, New York.

Young, P. (1994). Equity. Princeton University Press.

Zălinescu, C. (2002). Convex analysis in general vector spaces. World Scientific, London. 